\title{
A MATHEMATICAL MODEL FOR TWO DIMENSIONAL LOADING PROBLEM IN CROSS-DOCKING NETWORK DESIGN
}

\author{
İlker Küçükoğlu, Aslı Aksoy, Seval Ene and Nursel Öztürk \\ Department of Industrial Engineering, Uludag University, 16059, Gorukle, Bursa, \\ Turkey \\ ikucukoglu@uludag.edu.tr, asliaksoy@uludag.edu.tr, sevalene@uludag.edu.tr \\ nursel@uludag.edu.tr
}

\begin{abstract}
In this paper, the transportation problem of cross-docking network is taken into account. Cross-docking centers differ from distribution centers or warehouses in that the loads are transported from origins to destinations through cross-docking centers without storing them for a long time. Goods are unloaded from incoming trucks and reloaded immediately onto outgoing trucks in these cross-docking centers. This problem is formulated using a mixed integer programming with two-dimensional loading constraints. The two-dimensional shapes are applied for both trucks and loads in order to find exact capacity of each truck in basis of each product. The illustrative problems are solved and results show that the proposed model finds practical solutions with twodimensional loading constraints.
\end{abstract}

Key Words- Cross-docking, transportation problem, mathematical modeling

\section{INTRODUCTION}

Cross-docking is a logistics strategy recently used by many companies in order to reduce inventory and improve customer satisfaction. Products are transferred from incoming trucks to outgoing trucks without storing them for a long time (generally less than 24 hours) in these centers. This strategy provides different advantages compared with traditional distribution centers: the consolidation of shipments, a shorter delivery lead time, the reduction of costs, improved customer service, fewer overstocks, etc. [1]. As a result of these advantages, cross-docking has become an interesting logistics strategy that can give companies important competitive benefits.

Considerable research on cross-docking has been investigated in the literature. These studies can be classified based on the problem type: location of cross-docks, crossdocking layout design, cross-docking networks, vehicle routing, dock door assignment, truck scheduling, storage and other issues. Some of these problems are more concerned about long term decisions (strategic or tactical), while others deal with short-term decisions (operational).

Cross-docking network problem consist of supplier, customer and cross-docking facility sets. Each set contains one or more locations and the aim is to determine the flow of goods from suppliers to customers through cross-docks in order to reduce the total transportation cost. Lim et al. [2] studied the cross-docking network problem by extending the traditional transshipment problem. The transshipment problem consists of a number of supplier, transshipment and demand nodes with the capacitated arcs. 
Moreover, supplier and customer time windows are considered to determine inventory holding costs in this study. Chen et al. [3] studied a similar problem in cross-docking network design. The noticeable differences between these studies are that supplies and demands are not allowed to split and different products can be considered. An integer programming formulation of the problem is provided and three heuristic algorithms are proposed as a solution approach. Musa et al. [4] evaluates the total cost as distinct from other studies by considering vehicle transportation costs. They formulated the problem with integer programming and proposed an ant colony optimization meta-heuristic algorithm to solve the problem. Ma et al. [5] studied similar problem by considering only one type of product and formulated the problem with time costs and truck setup costs. The authors proposed a solution approach which consists of two stage heuristics. Alpan et al. [6] studied the transshipment scheduling problem in a multiple inbound and outbound dock configuration. Direct shipping and inventory holding strategies are allowed in the problem. The objective is to find best schedule of transshipment operations. They proposed several heuristic algorithms to attain the solution. Miao et al. [7] considered the transshipment problem with soft and hard time windows constraints. They proposed two types of meta-heuristics algorithms: tabu search and genetic algorithm.

On the other hand, there are considerable numbers of study related with the truck loading or pallet loading in the literature. Chen et al. [8] proposed a binary mathematical model for two-dimensional pallet packing problem for non-uniform box sizes and multiple pallets. Zachariadis et al. [9] developed a tabu search meta-heuristic algorithm for vehicle routing problem with two-dimensional loading constraints and tested their algorithm on several benchmark instances. They achieved to several new best solutions. Fuellerer et al. [10] considered the vehicle routing problem with threedimensional loading constraints. They presented an ant colony optimization algorithm as a solution approach. Zachariadis et al. [11] introduced a new transportation problem called the pallet-packing vehicle routing problem and used tabu search based heuristic algorithm to solve problem. Finally, Leung et al. [12] used a simulated annealing metaheuristic algorithm for heterogeneous fleet vehicle routing problem with twodimensional loading constraints and tested with benchmark instances derived from the two-dimensional loading vehicle routing problem. However, only a paper which considers truck loading in cross-docking network problem could be found to the best of our knowledge. Charkhgard \& Tabar [13] considered three dimensional products and truck shapes to find exact capacity of the trucks. But only one type of trucks and cubic products are assumed in their study. Moreover, there is no decision variable for truck loading plan.

In this study, we consider the two-dimensional loading problem in cross-docking network design. The two-dimensional shapes are applied for both trucks and loads in order to find exact capacity of each truck in basis of each product. The objective is to find the best network flow routes and truck loading plan decisions that minimize the total transportation cost. 
The rest of the paper is organized as follows. In Section 2, problem definition and mixed integer mathematical model is presented. In Section 3, the illustrative problems and their solutions are given. Finally, Section 4 concludes the study.

\section{PROBLEM DESCRIPTION}

In this paper, cross-docking network is considered for $S$ suppliers (origins), $D$ customers (destinations) and $C$ cross-docking facilities. The products flow from origins to destinations through cross-docks according to customers demand. Each product of suppliers is loaded into incoming and outgoing trucks by considering its destination. Thus, the objective is to find the best transshipment plan regarding the two dimensional truck loading operations in order to minimize total transportation costs. Musa et al. [4] and Charkhgard \& Tabar [8] considered some assumptions in their mathematical models. In this paper we have accepted several of them and enhanced our model with the following new conditions:

- Directly shipping is not allowed from suppliers to destinations.

- Truck capacity is taken into account with dimensional constraints on the contrary of weight or amount of load.

- Loads and trucks are considered as rectangular shapes.

- The trucks may have different sizes. Thus, loading plans are affected by the truck choices. Moreover each product to be sent from different origins to different destinations may have different sizes.

- The transportation costs are related with only travelled distances among the locations.

The concept of the two-dimensional loading problem in cross-docking network is depicted in Figure 1, which illustrates an example of two suppliers, two cross-dock facilities and three customers.

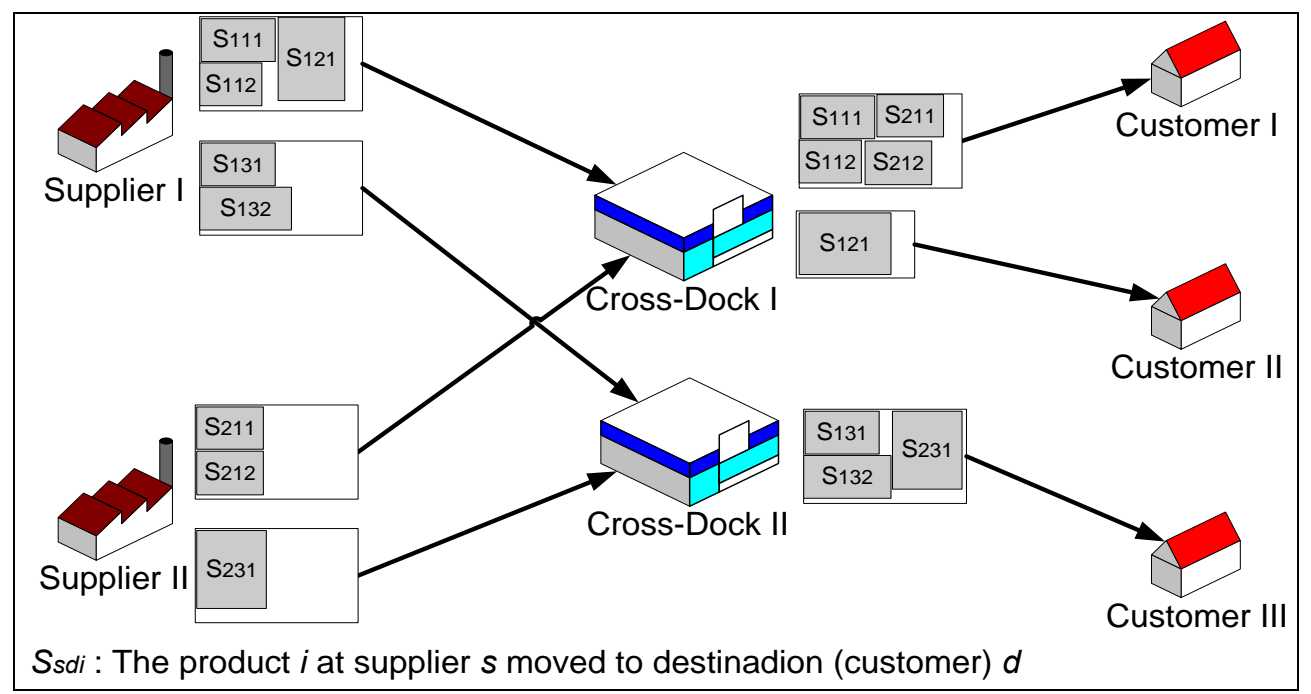

Figure 1. An illustrative example of described problem 
According to assumptions described above the problem can be formulated as mixed integer programming using the following notations:

$S \quad$ : The set of origins

$D \quad$ : The set of destinations

C : The set of cross-docks

$V_{-} S_{s}:$ Number of trucks at origin $s$, where $\forall s \in S$

$V \_C_{c}:$ Number of trucks at cross-dock $c$, where $\forall c \in C$

$F_{s d} \quad:$ Number of boxes flows at origin $s$ to destination $d$, where $\forall s \in S, \forall d \in D$

$L \_S_{s m}:$ Length of truck $m$ at origin $s$, where $\forall s \in S, \forall m \in\left\{1, \ldots, V \_S_{s}\right\}$

$L_{-} C_{c n}$ : Length of truck $n$ at cross-dock $c$, where $\forall c \in C, \forall n \in\left\{1, \ldots, V \_C{ }_{c}\right\}$

$W \_S_{s m}$ : Width of truck $m$ at origin $s$, where $\forall s \in S, \forall m \in\left\{1, \ldots, V_{-} S_{s}\right\}$

$W \_C_{c n}$ : Width of truck $n$ at cross-dock $c$, where $\forall c \in C, \forall n \in\left\{1, \ldots, V \_C_{c}\right\}$

$p_{\_} s_{s c}$ : Cost of a truck from origin $s$ to cross-dock $c$, where $\forall s \in S, \forall c \in C$

$p_{\_} c_{c d}$ : Cost of a truck from cross-dock $c$ to destination $d$, where $\forall c \in C, \forall d \in D$

$l_{s d i}:$ Length of box $i$ at origin $s$ for destination $d$, where $\forall s \in S, \forall d \in D$, $\forall i \in\left\{1, \ldots, F_{\text {sd }}\right\}$

$w_{s d i}:$ Width of box $i$ at origin $s$ for destination $d$, where $\forall s \in S, \forall d \in D$, $\forall i \in\left\{1, \ldots, F_{s d}\right\}$

$M \quad$ : Arbitrarily large constant

The variables are:

$x \_s_{s d i}^{m c} \quad: x$ coordinate of the south west corner of box $i$ in truck $m$ from origin $s$ to cross-dock $c$ related to destination $d$, where $\forall s \in S, \forall d \in D$, $\forall c \in C, \forall i \in\left\{1, \ldots, F_{s d}\right\}, \forall m \in\left\{1, \ldots, V \_S_{s}\right\}$

$x \_c_{s d i}^{n c} \quad: x$ coordinate of the south west corner of box $i$ in truck $n$ from crossdock $c$ to destination $d$ related to origin $s$, where $\forall s \in S, \forall d \in D$, $\forall c \in C, \forall i \in\left\{1, \ldots, F_{s d}\right\}, \forall n \in\left\{1, \ldots, V \_C_{c}\right\}$

$y_{-} s_{s d i}^{m c} \quad: y$ coordinate of the south west corner of box $i$ in truck $m$ from origin $s$ to cross-dock $c$ related to destination $d$, where $\forall s \in S, \forall d \in D$, $\forall c \in C, \forall i \in\left\{1, \ldots, F_{s d}\right\}, \forall m \in\left\{1, \ldots, V \_S_{s}\right\}$ 
$y_{-} c_{s d i}^{n c}$

$v_{-} s_{s}^{m c}$

$v_{-} c_{c}^{n d}$

$z-s_{s d i}^{m c}$

$z_{-} c_{s d i}^{n c}$

$\alpha \_s_{s d o i f}^{m c}$

$\beta \_s_{\text {sdoif }}^{m c}$

$\gamma_{-} s_{\text {sdoif }}^{m c}$

$\delta_{-} s_{\text {sdoif }}^{m c}$

$\alpha \_c_{\text {sudif }}^{n c}$
: $y$ coordinate of the south west corner of box $i$ in truck $n$ from crossdock $c$ to destination $d$ related to origin $s$, where $\forall s \in S, \forall d \in D$, $\forall c \in C, \forall i \in\left\{1, \ldots, F_{s d}\right\}, \forall n \in\left\{1, \ldots, V_{-} C_{c}\right\}$

: is a binary variable and equal to 1 if truck $m$ is used for cross-dock $c$ at origin $s$, otherwise it is equal to 0 , where $\forall s \in S, \forall c \in C$, $\forall m \in\left\{1, \ldots, V_{-} S_{s}\right\}$

: is a binary variable and equal to 1 if truck $n$ is used for destination $d$ at cross-dock $c$, otherwise it is equal to 0 , where $\forall d \in D, \forall c \in C$, $\forall n \in\left\{1, \ldots, V_{-} C_{c}\right\}$

: is a binary variable and equal to 1 if box $i$ of origin $s$ related to destination $d$ is shipped from origin $s$ to cross-dock $c$ with truck $m$, otherwise it is equal to 0 , where $\forall s \in S, \forall d \in D, \forall c \in C$, $\forall i \in\left\{1, \ldots, F_{s d}\right\}, \forall m \in\left\{1, \ldots, V_{-} S_{s}\right\}$

: is a binary variable and equal to 1 if box $i$ of origin $s$ related to destination $d$ is shipped from cross-dock $c$ to destination $d$ with truck $n$, otherwise it is equal to 0 , where $\forall s \in S, \forall d \in D, \forall c \in C$, $\forall i \in\left\{1, \ldots, F_{s d}\right\}, \forall n \in\left\{1, \ldots, V{ }_{-} C_{c}\right\}$

: is a binary variable and equal to 1 if box $i$ belongs to destination $d$ is on the left side of box $f$ belongs to destination $o$ in truck $m$ which flows from origin $s$ to cross-dock $c$, otherwise it is equal to 0 , where $\forall s \in S$, $\forall d, o \in D, \forall c \in C, \forall i \in\left\{1, \ldots, F_{s d}\right\}, \forall f \in\left\{1, \ldots, F_{s o}\right\}, \forall m \in\left\{1, \ldots, V_{-} S_{s}\right\}$

: is a binary variable and equal to 1 if box $i$ belongs to destination $d$ is on the right side of box $f$ belongs to destination $o$ in truck $m$ which flows from origin $s$ to cross-dock $c$, otherwise it is equal to 0 , where $\forall s \in S$, $\forall d, o \in D, \forall c \in C, \forall i \in\left\{1, \ldots, F_{s d}\right\}, \forall f \in\left\{1, \ldots, F_{s o}\right\}, \forall m \in\left\{1, \ldots, V_{-} S_{s}\right\}$

: is a binary variable and equal to 1 if box $i$ belongs to destination $d$ is on the bottom side of box $f$ belongs to destination $o$ in truck $m$ which flows from origin $s$ to cross-dock $c$, otherwise it is equal to 0 , where $\forall s \in S$, $\forall d, o \in D, \forall c \in C, \forall i \in\left\{1, \ldots, F_{s d}\right\}, \forall f \in\left\{1, \ldots, F_{s o}\right\}, \forall m \in\left\{1, \ldots, V_{-} S_{s}\right\}$

: is a binary variable and equal to 1 if box $i$ belongs to destination $d$ is on the top side of box $f$ belongs to destination $o$ in truck $m$ which flows from origin $s$ to cross-dock $c$, otherwise it is equal to 0 , where $\forall s \in S$, $\forall d, o \in D, \forall c \in C, \forall i \in\left\{1, \ldots, F_{s d}\right\}, \forall f \in\left\{1, \ldots, F_{s o}\right\}, \forall m \in\left\{1, \ldots, V_{-} S_{s}\right\}$

: is a binary variable and equal to 1 if box $i$ loaded from origin $s$ is on the left side of box $f$ loaded from origin $u$ in truck $n$ which flows from crossdock $c$ to destination $d$, otherwise it is equal to 0 , where $\forall s, u \in S$, $\forall d \in D, \forall c \in C, \forall i \in\left\{1, \ldots, F_{s d}\right\}, \forall f \in\left\{1, \ldots, F_{u d}\right\}, \forall n \in\left\{1, \ldots, V_{-} C_{c}\right\}$ 
$\beta \_c_{\text {sudif }}^{n c} \quad:$ is a binary variable and equal to 1 if box $i$ loaded from origin $s$ is on the right side of box $f$ loaded from origin $u$ in truck $n$ which flows from cross-dock $c$ to destination $d$, otherwise it is equal to 0 , where $\forall s, u \in S$, $\forall d \in D, \forall c \in C, \forall i \in\left\{1, \ldots, F_{s d}\right\}, \forall f \in\left\{1, \ldots, F_{u d}\right\}, \forall n \in\left\{1, \ldots, V \_C_{c}\right\}$

$\gamma_{-} c_{\text {sudif }}^{n c} \quad:$ is a binary variable and equal to 1 if box $i$ loaded from origin $s$ is on the bottom side of box $f$ loaded from origin $u$ in truck $n$ which flows from cross-dock $c$ to destination $d$, otherwise it is equal to 0 , where $\forall s, u \in S$, $\forall d \in D, \forall c \in C, \forall i \in\left\{1, \ldots, F_{s d}\right\}, \forall f \in\left\{1, \ldots, F_{u d}\right\}, \forall n \in\left\{1, \ldots, V \_C_{c}\right\}$

$\delta \_c_{s u d i f}^{n c} \quad:$ is a binary variable and equal to 1 if box $i$ loaded from origin $s$ is on the top side of box $f$ loaded from origin $u$ in truck $n$ which flows from crossdock $c$ to destination $d$, otherwise it is equal to 0 , where $\forall s, u \in S$, $\forall d \in D, \forall c \in C, \forall i \in\left\{1, \ldots, F_{s d}\right\}, \forall f \in\left\{1, \ldots, F_{u d}\right\}, \forall n \in\left\{1, \ldots, V_{-} C_{c}\right\}$

The mathematical model can be formulated as follows:

$\operatorname{Min} \sum_{s \in S} \sum_{c \in C} \sum_{m=1}^{V-S_{s}} p_{-} s_{s c} \cdot v_{-} s_{s}^{m c}+\sum_{c \in C} \sum_{d \in D}^{V} \sum_{n=1}^{L_{-} C_{c}} p_{-} c_{c d} \cdot v_{-} c_{c}^{n d}$

s.t.

$$
\begin{aligned}
& \sum_{c \in C} \sum_{m=1}^{V-S_{s}} z_{-} s_{s d i}^{m c}=1 \quad \forall s \in S, \quad \forall d \in D, \quad \forall i \in\left\{1, \ldots, F_{s d}\right\} \\
& \sum_{c \in C} v_{-} s_{s}^{m c} \leq 1 \quad \forall s \in S, \quad \forall m \in\left\{1, \ldots, V_{-} S_{s}\right\} \\
& \sum_{d \in D} \sum_{i=1}^{F_{s d}} z_{-} s_{s d i}^{m c} \leq M \cdot v_{-} s_{s}^{m c} \quad \forall s \in S, \quad \forall c \in C, \quad \forall m \in\left\{1, \ldots, V_{-} S_{s}\right\} \\
& \sum_{c \in C} \sum_{n=1}^{V-C_{c}} z_{-} c_{s d i}^{n c}=1 \quad \forall s \in S, \quad \forall d \in D, \quad \forall i \in\left\{1, \ldots, F_{s d}\right\} \\
& \sum_{d \in D} v_{-} c_{c}^{n d} \leq 1 \quad \forall c \in C, \quad \forall n \in\left\{1, \ldots, V_{-} C_{c}\right\} \\
& \sum_{d \in D} \sum_{i=1}^{F_{s d}} z_{-} c_{s d i}^{n c} \leq M \cdot v_{-} c_{c}^{n d} \quad \forall d \in D, \quad \forall c \in C, \quad \forall n \in\left\{1, \ldots, V_{-} C_{c}\right\} \\
& \sum_{s \in S} \sum_{d \in D} \sum_{i=1}^{F_{s d}} \sum_{m=1}^{V \_} z_{-} s_{s d i}^{m c}=\sum_{s \in S} \sum_{d \in D} \sum_{i=1}^{F_{s d}} \sum_{n=1}^{V-C_{c}} z_{-} c_{s d i}^{n c} \quad \forall c \in C \\
& x_{-} s_{s d i}^{m c}+l_{s d i} \leq x_{-} s_{s o f}^{m c}+M \cdot\left(1-\alpha_{-} s_{s d o i f}^{m c}\right) \\
& \forall s \in S, \forall d, o \in D, \forall m \in\left\{1, \ldots, V_{-} S_{s}\right\}, \forall c \in C, \forall i \in\left\{1, \ldots, F_{s d}\right\}, \forall f \in\left\{1, \ldots, F_{s o}\right\} \\
& x_{-} s_{s o f}^{m c}+l_{s o f} \leq x_{-} s_{s d i}^{m c}+M \cdot\left(1-\beta_{-} s_{s d o i f}^{m c}\right) \\
& \forall s \in S, \forall d, o \in D, \forall m \in\left\{1, \ldots, V_{-} S_{s}\right\}, \forall c \in C, \forall i \in\left\{1, \ldots, F_{s d}\right\}, \forall f \in\left\{1, \ldots, F_{s o}\right\}
\end{aligned}
$$




$$
\begin{aligned}
& y_{-} s_{s d i}^{m c}+w_{s d i} \leq y_{-} s_{s o f}^{m c}+M \cdot\left(1-\gamma_{-} s_{s d o i f}^{m c}\right) \\
& \quad \forall s \in S, \forall d, o \in D, \forall m \in\left\{1, \ldots, V_{-} S_{s}\right\}, \forall c \in C, \forall i \in\left\{1, \ldots, F_{s d}\right\}, \forall f \in\left\{1, \ldots, F_{s o}\right\} \\
& y_{-} s_{s o f}^{m c}+w_{\text {sof }} \leq y_{-} s_{s d i}^{m c}+M \cdot\left(1-\delta_{-} s_{s d o i f}^{m c}\right) \\
& \quad \forall s \in S, \forall d, o \in D, \forall m \in\left\{1, \ldots, V_{-} S_{s}\right\}, \forall c \in C, \forall i \in\left\{1, \ldots, F_{s d}\right\}, \forall f \in\left\{1, \ldots, F_{s o}\right\}
\end{aligned}
$$

$$
\begin{aligned}
& \alpha_{-} s_{\text {sdoif }}^{m c}+\beta_{-} s_{\text {sdoif }}^{m c}+\gamma_{-} s_{\text {sdoif }}^{m c}+\delta_{-} s_{\text {sdoif }}^{m c} \geq z_{-} s_{\text {sdi }}^{m c}+z_{-} s_{s o f}^{m c}+1 \\
& \forall s \in S, \forall d, o \in D, \forall m \in\left\{1, \ldots, V_{-} S_{s}\right\}, \forall c \in C, \forall i \in\left\{1, \ldots, F_{s d}\right\}, \forall f \in\left\{1, \ldots, F_{s o}\right\} \\
& \sum_{c \in C} x_{-} s_{s d i}^{m c}+l_{s d i} \leq L_{-} S_{s m} s \in S, \forall d \in D, \forall m \in\left\{1, \ldots, V_{-} S_{s}\right\}, \forall i \in\left\{1, \ldots, F_{s d}\right\} \\
& \sum_{c \in C} y_{-} s_{s d i}^{m c}+w_{s d i} \leq W_{-} S_{s m} \forall s \in S, \forall d \in D, \forall m \in\left\{1, \ldots, V_{-} S_{s}\right\}, \forall i \in\left\{1, \ldots, F_{s d}\right\} \\
& x_{-} s_{s d i}^{m c}+y_{-} s_{s d i}^{m c} \leq M \cdot z_{-} s_{s d i}^{m c} \forall s \in S, \forall d \in D, \forall c \in C \forall, m \in\left\{1, \ldots, V_{-} S_{s}\right\}, \forall i \in\left\{1, \ldots, F_{s d}\right\} \\
& x_{-} c_{s d i}^{n c}+l_{s d i} \leq x_{-} c_{u d f}^{n c}+M \cdot\left(1-\alpha_{-} c_{\text {sudif }}^{n c}\right) \\
& \forall s, u \in S, \forall d \in D, \forall n \in\left\{1, \ldots, V_{-} C_{c}\right\}, \forall c \in C, \forall i \in\left\{1, \ldots, F_{s d}\right\}, \forall f \in\left\{1, \ldots, F_{u d}\right\} \\
& x_{-} c_{u d f}^{n c}+l_{u d f} \leq x_{-} c_{s d i}^{n c}+M \cdot\left(1-\beta_{-} c_{\text {sudif }}^{n c}\right) \\
& \forall s, u \in S, \forall d \in D, \forall n \in\left\{1, \ldots, V_{-} C_{c}\right\}, \forall c \in C, \forall i \in\left\{1, \ldots, F_{s d}\right\}, \forall f \in\left\{1, \ldots, F_{u d}\right\} \\
& y_{-} c_{s d i}^{n c}+w_{s d i} \leq y_{-} c_{u d f}^{n c}+M \cdot\left(1-\gamma_{-} c_{\text {sudif }}^{n c}\right) \\
& \forall s, u \in S, \forall d \in D, \forall n \in\left\{1, \ldots, V_{-} C_{c}\right\}, \forall c \in C, \forall i \in\left\{1, \ldots, F_{s d}\right\}, \forall f \in\left\{1, \ldots, F_{u d}\right\} \\
& y_{-} c_{u d f}^{n c}+w_{u d f} \leq y_{-} c_{s d i}^{n c}+M \cdot\left(1-\delta_{-} c_{\text {sudif }}^{n c}\right) \\
& \forall s, u \in S, \forall d \in D, \forall n \in\left\{1, \ldots, V_{-} C_{c}\right\}, \forall c \in C, \forall i \in\left\{1, \ldots, F_{s d}\right\}, \forall f \in\left\{1, \ldots, F_{u d}\right\} \\
& \alpha_{-} c_{\text {sudif }}^{n c}+\beta \beta_{-} c_{\text {sudif }}^{n c}+\gamma_{-} c_{\text {sudif }}^{n c}+\delta_{-} c_{\text {sudif }}^{n c} \geq z_{-} c_{\text {sdi }}^{n c}+z_{-} c_{\text {sof }}^{n c}+1 \\
& \forall s, u \in S, \forall d \in D, \forall n \in\left\{1, \ldots, V_{-} C_{c}\right\}, \forall c \in C, \forall i \in\left\{1, \ldots, F_{s d}\right\}, \forall f \in\left\{1, \ldots, F_{u d}\right\} \\
& x_{-} c_{s d i}^{n c}+l_{s d i} \leq L_{-} C_{c n} s \in S, \forall d \in D, \forall n \in\left\{1, \ldots, V_{-} C_{c}\right\}, \forall c \in C, \forall i \in\left\{1, \ldots, F_{s d}\right\} \\
& y_{-} c_{s d i}^{n c}+w_{s d i} \leq W_{-} C_{c n} \forall s \in S, \forall d \in D, \forall n \in\left\{1, \ldots, V_{-} C_{c}\right\}, \forall c \in C, \forall i \in\left\{1, \ldots, F_{s d}\right\} \\
& x_{-} c_{s d i}^{n c}+y_{-} c_{s d i}^{n c} \leq M \cdot z_{-} c_{s d i}^{n c} \forall s \in S, \forall d \in D, \forall n \in\left\{1, \ldots, V_{-} C_{c}\right\}, \forall c \in C, \forall i \in\left\{1, \ldots, F_{s d}\right\} \\
& v_{-} s_{s}^{m c}, v_{-} c_{c}^{n d}, z_{-} s_{s d i}^{m c}, z_{-} c_{s d i}^{n c}, \alpha_{-} s_{\text {sdoif }}^{m c}, \beta_{-} s_{s d o i f}^{m c}, \gamma_{-} s_{\text {sdoif }}^{m c}, \delta_{-} s_{s d o i f}^{m c}, \alpha_{-} c_{s u d i f}^{n c}, \beta_{-} c_{\text {sudif }}^{n c}, \gamma_{-} c_{\text {sudif }}^{n c}, \delta_{-} c_{\text {sudif }}^{n c}=\text { Binary } \\
& x_{-} s_{s d i}^{m c}, y_{-} s_{s d i}^{m c}, x_{-} c_{s d i}^{n c}, y_{-} c_{s d i}^{n c} \geq 0
\end{aligned}
$$

The objective (1) is to minimize the total transportation cost. The constraints consist of two parts: cross-docking consolidation (constraints (2) to (8)) and two dimensional truck loading (constraints (9) to (24)) decisions.

For the cross-docking consolidation decisions, constraint (2) and (5) assign the product to incoming and outgoing trucks and ensures that each product can be assigned only one incoming and outgoing truck respectively. Constraint (3) provides that each truck at origins can be sent at most one cross-docking facility and also constraint (6) maintains this condition at cross-docking trucks that each truck at cross-docking facilities can be 
sent at most one destination. Constraint (4) and (7) define that each product can be assigned to incoming or outgoing truck if the designated truck is used. Constraint (8) forces the product continuity at cross-docking facilities.

For the truck loading decisions, constraint (9) to (12) and constraint (17) to (20) provide that products do not overlap each other if a pair of products is in the same truck and it is determined by constraint (13) and constraint (21) according to incoming and outgoing transport respectively. Constraint (14) and (15) ensure that all the products loaded in an incoming truck fit within the dimensions of the truck. Likewise, constraint (22) and (23) ensure the same condition for outgoing trucks. If a truck is not used then there should not be any product loaded in it. This requirement is handled by constraint (16) for incoming trucks and constraint (24) for outgoing trucks.

\section{COMPUTATIONAL EXPERIMENTS}

Because cross-docking network design and two-dimensional truck loading problem have been not considered jointly before, there is no benchmark test set available. Therefore, we generated our own data sets randomly includes different scenarios to check the validity of the model. The test problems are described with four basic parameters $\left(S / C / D / F_{\max }\right)$ : the number of suppliers $S$, the number of cross-dock facilities $C$, the number of destinations $D$ and maximum flow amount in network $F_{\text {max }}$. Table 1 presents the rest of the parameter ranges used for randomly generated instances.

Table 1. Parameter ranges

\begin{tabular}{llll}
\hline Transportation cost ranges $\left(p_{-} s_{s c}, p_{-} c_{c d}\right)$ & $: U[50,150]$ & Truck width ranges $\left(W_{-} S_{s m}, W_{-} C_{c n}\right)$ & $: U[50,150]$ \\
Product dimension ranges $\left(w_{s d i}, l_{s d i}\right)$ & $: U[5,25]$ & Truck length ranges $\left(L_{-} S_{s m}, L_{-} C_{c n}: U[100,250]\right.$ \\
Flow ranges $\left(F_{s d}\right)$ & $: U\left[0, F_{\text {max }}\right]$ & Truck numbers $\left(V_{S_{S}}, V_{C_{c}}\right)$ & $: U[0,8]$ \\
\hline
\end{tabular}

We performed 10 different problem categories and each category contains five randomly generated problem instances. Instances are solved with CPLEX 11.1.1 on a Pentium ${ }^{\circledR} 2.2 \mathrm{GHz}$ CPU with $2.0 \mathrm{~GB}$ memory. Each output of the model is examined to verify solution. To illustrate the experiments, Table 2 includes a data set belongs to problem category 3/2/4/4 and Table 3 represents the optimal solution (objective function value $=944.0$ ) of the illustrative example. Each row describes the integrated product flow plan of a product in a network design and includes the product-vehicle assignment and product locations ( $x$ and $y$ coordinates) for incoming and outgoing trucks respectively. Also, assignment of the incoming trucks to cross-docking centers and assignment of the outgoing trucks to customers can be obtained by the table.

Table 2. A data set for 3/2/4/4 problem type

\begin{tabular}{llll}
\hline$V_{-} S_{s}$ & $=\{3,2,4\}$ & $V_{-} C_{c}$ & $=\{3,2\}$ \\
$L_{-} S_{s m}$ & $=\{50,130,80,110,70,90,110,140,60\}$ & $L_{-} C_{c n}$ & $=\{50,140,70,100,90\}$ \\
$W_{-} S_{s m}$ & $=\{110,160,240,190,140,110,200,230,120\}$ & $W_{-} C_{c n}$ & $=\{130,180,110,170,160\}$ \\
$p_{-} s_{s c}$ & $=\{136,129,87,146,137,56\}$ & $p_{-} c_{c d}$ & $=\{145,86,102,127,55,109,97,80\}$ \\
$F_{s d}$ & $=\{3,2,2,1,1,3,0,3,3,3,0,2\}$ & & \\
$l_{s d i}$ & $=\{18,11,21,25,10,25,16,25,5,7,21,6,13,24,13,8,18,13,12,9,17,14,10\}$ \\
$w_{s d i}$ & $=\{17,10,22,17,23,19,10,7,19,17,7,11,11,11,25,11,8,13,19,18,9,7,23\}$
\end{tabular}


Table 3. Solution of the illustrative example

\begin{tabular}{|c|c|c|c|c|c|c|c|c|c|c|}
\hline \multirow[b]{2}{*}{ \# } & \multirow[b]{2}{*}{$\begin{array}{l}\text { Supplier } \\
\text { (s) }\end{array}$} & \multirow[b]{2}{*}{$\begin{array}{c}\text { Customer } \\
(d)\end{array}$} & \multirow[b]{2}{*}{$\begin{array}{l}\text { Product } \\
\text { (i) }\end{array}$} & \multirow[b]{2}{*}{$\begin{array}{c}\text { Cross_Dock } \\
(c)\end{array}$} & \multicolumn{3}{|c|}{ Incoming Trucks } & \multicolumn{3}{|c|}{ Outgoing Trucks } \\
\hline & & & & & $\begin{array}{c}\text { Vehicle } \\
(k)\end{array}$ & $\begin{array}{c}\text { x_coordinate } \\
(x)\end{array}$ & $\begin{array}{c}\text { y_coordinate } \\
(y)\end{array}$ & $\begin{array}{c}\text { Vehicle } \\
(k)\end{array}$ & $\begin{array}{c}\text { x_coordinate } \\
(x)\end{array}$ & $\begin{array}{c}\text { y_coordinate } \\
(y)\end{array}$ \\
\hline 1 & 1 & 1 & 1 & 1 & 1 & 3 & 52 & 2 & 29 & 6 \\
\hline 2 & 1 & 1 & 2 & 1 & 1 & 0 & 69 & 2 & 0 & 0 \\
\hline 3 & 1 & 1 & 3 & 1 & 1 & 0 & 30 & 2 & 119 & 42 \\
\hline 4 & 1 & 2 & 1 & 1 & 1 & 21 & 7 & 3 & 45 & 0 \\
\hline 5 & 1 & 2 & 2 & 1 & 1 & 0 & 7 & 3 & 21 & 19 \\
\hline 6 & 1 & 3 & 1 & 2 & 3 & 0 & 10 & 1 & 0 & 0 \\
\hline 7 & 1 & 3 & 2 & 2 & 3 & 0 & 0 & 1 & 25 & 0 \\
\hline 8 & 1 & 4 & 1 & 1 & 1 & 21 & 0 & 1 & 0 & 0 \\
\hline 9 & 2 & 1 & 1 & 1 & 1 & 37 & 0 & 2 & 11 & 64 \\
\hline 10 & 2 & 2 & 1 & 1 & 1 & 30 & 11 & 3 & 21 & 2 \\
\hline 11 & 2 & 2 & 2 & 1 & 1 & 42 & 28 & 3 & 0 & 53 \\
\hline 12 & 2 & 2 & 3 & 1 & 1 & 0 & 0 & 3 & 17 & 42 \\
\hline 13 & 2 & 4 & 1 & 1 & 1 & 55 & 0 & 1 & 13 & 119 \\
\hline 14 & 2 & 4 & 2 & 1 & 1 & 6 & 17 & 1 & 26 & 32 \\
\hline 15 & 2 & 4 & 3 & 1 & 1 & 42 & 35 & 1 & 0 & 7 \\
\hline 16 & 3 & 1 & 1 & 1 & 2 & 0 & 74 & 2 & 0 & 31 \\
\hline 17 & 3 & 1 & 2 & 1 & 2 & 20 & 66 & 2 & 0 & 23 \\
\hline 18 & 3 & 1 & 3 & 1 & 2 & 46 & 19 & 2 & 16 & 10 \\
\hline 19 & 3 & 2 & 1 & 1 & 2 & 8 & 0 & 3 & 5 & 0 \\
\hline 20 & 3 & 2 & 2 & 1 & 2 & 20 & 32 & 3 & 17 & 92 \\
\hline 21 & 3 & 2 & 3 & 1 & 2 & 29 & 57 & 3 & 0 & 60 \\
\hline 22 & 3 & 4 & 1 & 1 & 2 & 59 & 50 & 1 & 0 & 32 \\
\hline 23 & 3 & 4 & 2 & 1 & 2 & 73 & 0 & 1 & 13 & 9 \\
\hline
\end{tabular}

Table 4 presents the results of all instances. The first column of each instance is the objective function value of the optimum solution and the second column is the computational time (in second) of the CPLEX. It is clearly seen from the table that an optimum solution could be found for each instance by the proposed model. On the other hand, computational times show that CPLEX takes less than 10 minutes (without the run time limitation) to reach optimum solution for each problem. Moreover, the average computational time of the instances is 2.5 minutes and generally solutions could be found in one minute. Consequently computational results validate the capability of the proposed model.

Table 4. Results of the instances

\begin{tabular}{|c|c|c|c|c|c|c|c|c|c|c|c|c|}
\hline \multirow{2}{*}{ Problem } & \multicolumn{2}{|c|}{ Instance I } & \multicolumn{2}{|c|}{ Instance II } & \multicolumn{2}{|c|}{ Instance III } & \multicolumn{2}{|c|}{ Instance IV } & \multicolumn{2}{|c|}{ Instance V } & \multicolumn{2}{|c|}{ Average } \\
\hline & $\overline{\mathrm{OFV}}$ & Time(s) & OFV & Time(s) & OFV & Time(s) & $\overline{\mathrm{OFV}}$ & Time(s) & OFV & Time(s) & OFV & Time(s) \\
\hline $1 / 1 / 5 / 15$ & 611 & 240.0 & 522 & 225.0 & 613 & 188.0 & 706 & 457.0 & 652 & 225.0 & 620.8 & $\overline{267.0}$ \\
\hline $2 / 1 / 2 / 8$ & 384 & 0.2 & 325 & 0.2 & 366 & 0.3 & 441 & 0.1 & 431 & 0.2 & 389.4 & 0.2 \\
\hline $2 / 2 / 6 / 5$ & 933 & 217.0 & 717 & 914.0 & 766 & 395.0 & 918 & 535.0 & 936 & 116.0 & 854.0 & 435.4 \\
\hline $3 / 2 / 4 / 4$ & 1014 & 33.5 & 997 & 7.0 & 966 & 27.0 & 940 & 21.1 & 619 & 3.7 & 907.2 & 18.4 \\
\hline $3 / 4 / 2 / 6$ & 941 & 47.3 & 744 & 126.0 & 1014 & 43.5 & 793 & 104.0 & 965 & 194.0 & 891.4 & 103.0 \\
\hline $4 / 1 / 2 / 10$ & 663 & 24.0 & 520 & 2.8 & 572 & 12.0 & 588 & 5.1 & 544 & 355.0 & 577.4 & 79.8 \\
\hline $5 / 5 / 3 / 5$ & 721 & 62.0 & 761 & 18.2 & 751 & 21.1 & 854 & 45.9 & 686 & 23.9 & 754.6 & 34.2 \\
\hline $6 / 2 / 3 / 3$ & 1439 & 351.0 & 1137 & 51.5 & 1283 & 56.8 & 1304 & 374.0 & 1247 & 200.0 & 1282.0 & 206.6 \\
\hline $7 / 2 / 2 / 3$ & 889 & 4.9 & 1051 & 0.9 & 1544 & 1.3 & 1166 & 1.3 & 1145 & 1.0 & 1159.0 & 1.9 \\
\hline $9 / 2 / 4 / 2$ & 2023 & 505.0 & 1924 & 261.0 & 1789 & 29.8 & 2066 & 177.0 & 2150 & 79.0 & 1990.4 & 210.4 \\
\hline
\end{tabular}

\section{CONCLUSION AND FUTURE WORK}

In this work, the two-dimensional truck loading problem was studied first time for cross-docking network design in order to minimize the total transportation cost. Thus, 
loading and transportation operations can be carried out more realistic and applicable in real life. Problem is formulated with the mixed integer mathematical model. Proposed model is tested with several randomly generated instances and results are examined to verify solutions. Results show that our model verifies its capability. As a result, this model able to find practical solutions to cross-docking operations. For further work on this problem is to find a powerful meta-heuristic algorithm to find effective solutions on large scale problems.

Acknowledgements-This work was supported by The Commission of Scientific Research Projects of Uludag University, Project number KUAP(M)-2012/53.

\section{REFERENCES}

1. J.V. Belle, P. Valckenaers, D. Cattrysse, Cross-docking: State of the art, Omega 40(6), $827-846,2012$.

2. A. Lim, Z. Miao, B. Rodrigues, Z. Xu, Transshipment through crossdocks with inventory and time windows, Naval Research Logistics 52(8), 724 - 733, 2005.

3. P. Chen, Y. Guo, A. Lim, B. Rodrigues, Multiple crossdocks with inventory and time windows, Computers \& Operations Research 33, 43 - 63, 2006.

4. R. Musa, J. Arnaout, H. Jung, Ant colony optimization algorithm to solve the transportation problem of cross-docking network, Computers \& Industrial Engineering 59, 85 - 92, 2010.

5. H. Ma, Z. Miao, A. Lim, B. Rodrigues, Crossdocking distribution networks with setup cost and time windows constraint, Omega 39, 64 - 72, 2011.

6. G. Alpan, A. Ladier, R. Larbi, B. Penz, Heuristic solutions for transshipment problems in a multiple door cross docking warehouse, Computers \& Industrial Engineering 61, 402 - 408, 2011.

7. Z. Miao, F. Yang, K. Fu, D. Xu, Transshipment service through crossdocks with both soft and hard time windows, Annals of Operations Research 192, 21 - 47, 2012.

8. C. S. Chen, S. Sarin, B. Ram, The pallet packing problem for non-uniform box sizes, International Journal of Production Research 29(10), 1963 - 1968, 1991.

9. E. E. Zachariadis, C.D. Tarantilis, C.T. Kiranoudis, A guided tabu search for the vehicle routing problem with two-dimensional loading constraints, European Journal of Operational Research 195, 729 - 743, 2009.

10. G. Fuellerer, K.F. Doerner, R.H. Hartl, M. Iori, Metaheuristics for vehicle routing problems with three-dimensional loading constraints, European Journal of Operational Research 201, 751 - 759, 2010.

11. E. E. Zachariadis, C.D. Tarantilis, C.T. Kiranoudis, The pallet-packing vehicle routing problem, Transportation Science 46(3), 341 - 358, 2012.

12. S. C. H. Leung, Z. Zhang, D. Zhang, X. Hua, M.K. Lim, A meta-heuristic algorithm for heterogeneous fleet vehicle routing problems with two-dimensional loading constraints, European Journal of Operational Research 225, 199 - 210, 2013.

13. H. Charkhgard, A.A.Y. Tabar, Transportation problem of cross-docking network with three-dimensional trucks, African Journal of Business Management 5(22), $9297-9303,2011$. 